\title{
Incidence of avascular necrosis following biceps tenodesis during proximal humerus open reduction and internal fixation
}

\author{
Kunal Mohan, Justin Matthias Hintze, David Morrissey, Diarmuid Molony \\ From the Department of Trauma \& Orthopaedics, Tallaght University Hospital, Dublin D24NROA, Ireland
}

\begin{abstract}
Avascular necrosis (AVN) may occur in up to $77 \%$ of proximal humeral fractures and can cause fixation failure. Risk factors include fracture position, calcar length and medial hinge integrity. We routinely perform intra-articular biceps tenotomy with tenodesis at the level of pectoralis major to facilitate fragment identification and potentially ameliorate post-operative pain relief. Concern exists that tenotomising the biceps damages the adjacent arcuate artery, potentially increasing the rate of $\mathrm{AVN}$. The purpose of this study was to evaluate whether biceps tenodesis is associated with an increased risk of radiographically evident humeral head AVN. 61 fractures surgically treated over a 52-month period were retrospectively reviewed and radiographically assessed in accordance with Neer's classification, calcar-length and medial hinge integrity.

40, 20 and 1 were four-, three- and two-part fractures respectively. 37 had a calcar-length less than $8 \mathrm{~mm}$ and 26 suffered loss of the medial hinge. The median radiographic follow-up was 23 months. There was radiographic evidence of humeral head $A V N$ in only one case, comparing favourably to rates quoted in current literature.

In our experience, intra-articular biceps tenotomy with the deltopectoral approach was thus not associated with a significantly increased risk of humeral head $\mathrm{AVN}$, even in complex four-part fractures.
\end{abstract}

Keywords : proximal humerus ; open reduction internal fixation ; avascular necrosis ; biceps ; tenodesis ; deltopectoral.

No benefits or funds were received in support of this study. None of the authors have a conflict of interest.

\section{INTRODUCTION}

Proximal humerus fractures are common, representing approximately five percent of all fractures presenting to the emergency department (1). These fractures are particularly prominent in an elderly osteoporotic patient group, with over seventy percent of proximal humerus fractures occurring in those sixty years of age or older (2). The overall incidence of proximal humerus fractures is increasing at a rate of up to $15 \%$ per year (3). They are up to four times more common in females than males (4), and occur most frequently as a result of low-energy trauma in an ageing osteoporotic patient group $(5,6)$. Comorbid and inactive, frail patients with pre-existing limitations to their activities of daily living have been found to be at higher risk of suffering these fractures (4). Proximal humerus fractures can result in significant morbidity (7) and functional impairment (8) in the elderly patient, hence

- Kunal Mohan*,

- Justin Matthias Hintze*,

- David Morrissey,

- Diarmuid Molony

Department of Trauma \& Orthopaedics, Tallaght University

Hospital, Dublin D24NROA, Ireland.

* Co-first authors.

Correspondence : Dr. Kunal Mohan, Department of Trauma \& Orthopaedics, Tallaght University Hospital, Dublin D24NROA, Ireland. Phone : +353-14142000.

Email : kmohan@tcd.ie

- 2021, Acta Orthopædica Belgica. 
making appropriate evaluation and management of paramount importance.

Radiographic evaluation consists of anteroposterior, scapular-Y and axillary views, often followed by computed tomography (CT) with threedimensional reconstruction (9), allowing for thorough evaluation of fracture anatomy (9) as well as objective fracture classification. Classification, while challenging (6), plays an important role in assessment, as it helps clinical decision making on subsequent management (6). A commonly used system for describing proximal humerus fractures is Neer's Classification (6,10), which divides the proximal humerus into 4 parts and describes fractures based on the amount of displaced segments, defining a displaced segment is defined as greater than $1 \mathrm{~cm}$ displacement or 45 degree angulation. Alternative classifications include the AO (11) and Hertel (12) systems.

The majority of proximal humerus fractures are either undisplaced or minimally displaced and often managed nonoperatively (13). These patients are typically associated with good functional outcomes and low complication rates (14). Surgical management is usually considered in cases of complex, multifragmentary, displaced fractures or fracture dislocations (6) in the independent, highfunctioning and healthy patient (15), especially when assessed by specialist shoulder and elbow surgeons (15). Surgical options are numerous, broadly classified into reconstructive options or arthroplasty (6). Technical factors such as fracture pattern, bone quality and rotator cuff integrity as well as patient factors such as age, functional and comorbid status help dictate selection of operative modality for each individual fracture (16).

A factor complicating management is the risk of post-traumatic avascular necrosis (AVN) (17). Humeral head AVN is a devastating complication that can lead to significant patient morbidity and limitation (18). Underlying fracture characteristics such as the degree of displacement and number of fracture parts correlated with the risk of AVN, with four-part fractures carrying the greatest risk $(10,19)$. Other suggested risk factors for AVN include the length of posteromedial metaphyseal fracture extension, disruption of the medial hinge and fractures involving the anatomic neck (12), as well as underlying patient factors. Direct injury to the axillary artery and its articular branches following proximal humerus fracture itself is rare, occurring only in $0.09 \%$ of patients (20). It has been suggested that prompt fracture reduction helps reduce the incidence of post-operative AVN in those managed surgically (18).

Open reduction and internal fixation (ORIF) with plate osteosynthesis is a surgical strategy often considered in those with displaced two or three part fractures, with the objective of the operating surgeon being to achieve adequate reduction and fixation, in an attempt to preserve native anatomy and optimise postoperative function (17). To achieve this, a number of intraoperative techniques can be used as per the surgeon's preference. Two primary approaches exist, namely deltopectoral and anterolateral (or deltoid-splitting) (17). The deltopectoral approach is the most commonly used (21), making it an attractive option as it offers wide exposure, while also following an internervous plane between deltoid and pectoralis major (17). However, this approach can limit exposure to the lateral and posterior aspects of the proximal humerus (21), which in turn can affect fracture visualisation and reduction.

To help combat this, we routinely perform an intra-articular tenotomy of the long head of biceps tendon (LHBT) during deltopectoral approach to proximal humerus ORIF, prior to tenodesis at the level of pectoralis major. This is performed so to facilitate fragment identification and aid fracture reduction, particularly in regards to the humeral head segment, as well as to potentially reduce postoperative pain due to potential LHBT damage or through entrapment in fracture callus at the level of the surgical neck (22). Although both LHBT tenotomy alone and additional tenodesis are viable strategies, patient satisfaction has been shown to be higher in those following tenodesis, particularly in the more elderly patient, thus further strengthening the argument for performing both procedures intraoperatively (23).

While LHBT tenotomy and tenodesis helps in fracture visualisation, it has been postulated that routinely tenotomising the LHBT may disrupt the 
blood supply to the proximal humerus. It has been suggested that the proximal humerus is significantly perfused by the anterolateral ascending branch of the anterior circumflex artery (24), which runs parallel to the LHBT in the bicipital groove before forming the arcuate artery. It has been suggested that this branch of the anterior circumflex humeral artery is at risk of damage during LHBT tenotomy and tenoodesis, which may theoretically disrupt perfusion to the proximal humerus and predispose it to AVN and its associated complications (25).

The objective of our study was hence to evaluate whether routine LHBT tenotomy and tenodesis during proximal humerus ORIF via deltopectoral approach is associated with an increased risk of humeral head AVN through comparison with reported rates in the literature.

\section{MATERIALS AND METHODS}

A retrospective, observational study of proximal humerus fractures undergoing open reduction and internal fixation by a single specialist senior upper limb consultant surgeon across two centres between January 2011 and April 2015 was performed. Patients were identified through assessment of a prospectively maintained surgical logbook of the primary surgeon, which recorded a patient's medical record number, demographics, hospital location and underlying systemic status at time of the index operation. Proximal humerus ORIF performed in circumstances of primary trauma via deltopectoral approach with intraoperative biceps tenodesis were deemed suitable for inclusion. Those performed in the setting of revision, malignancy or those operated upon via an deltoid-splitting approach were excluded.

Demographics including age and gender were recorded for each of the patients suitable for inclusion. Patient's underlying systemic status was reflected by the American Society of Anaesthesiologists Physical Status Scale (ASA-PS), a validated marker of patients preoperative health status (26). The type of fixation used for proximal humerus osteosynthesis in each individual fracture was also recorded.
Assessment of included patients consisted primarily of radiographic evaluation, assessed through utilisation of the McKesson Radiology ${ }^{\mathrm{TM}}$ picture archiving and communication (PACS) system. Preoperative radiographs were evaluated and classified as per Neer's Classification (10), with additional radiographic measurement of calcar length and medial hinge integrity performed for each individual fracture performed by two independent reviewers each involved in this study. Calcar length was calculated through obtaining the average of each individual reviewer's measurements and consensus in regards calcar integrity was achieved in each case between both reviewers following individual review in case of discordance.

Evaluation of the incidence of postoperative AVN of the humeral head following proximal humerus ORIF with intraoperative biceps tenodesis was the primary outcome of this study. This was assessed through evaluation of plain postoperative radiographs by the same two independent reviewers as those evaluating the preoperative radiographs. Plain radiographs with either dedicated shoulder views, or imaging of adjacent structures were deemed suitable for assessment, provided they allowed for full views of the relevant humeral head as well as lesser and greater tuberosities. The most recent radiograph fulfilling these criteria at time of assessment was used for evaluation of the presence of AVN.

The criteria for determining humeral head AVN on plain radiograph were extrapolated from the characteristics initially described by Ficat and Arlet in relation to proximal femoral AVN $(27,28)$, which have since been adopted as radiological evidence of AVN. Each radiograph was hence evaluated for the presence of subchondral sclerosis, presence of radiolucent cysts, diffuse osteopenia, as well as more pathognomonic imaging features of AVN such as a "crescent line," indicative of subchondral fracture, alteration in joint space and flattening of the humeral head (28), with a radiograph deemed as displaying AVN should any of these pathognomonic features be present. An evaluation of the incidence of tuberosity AVN was also performed at time of review. 


\section{RESULTS}

88 proximal humerus ORIFs were performed over a 52 month period across two centres, of which 61 were deemed suitable for inclusion for eventual analysis. These were undertaken in 60 patients, with one patient suffering from bilateral proximal humerus fractures during a single index injury, requiring subsequent bilateral ORIF on the same date. Of those included, 78\% were female (47/60) and $22 \%$ male $(13 / 60)$, with an overall mean age of 64 years (Standard Deviation $(\mathrm{SD})=13$ ) (Table I). $42 \%(25 / 60)$ of patients presented without any comorbidities (ASA I) at time of the index operation, with 47\% (28/60) and 11\% (7/60) presenting with mild (ASA II) and severe (ASA III) systemic disease, respectively (Table II).

Upon radiographic evaluation of all included fractures as per Neer's Classification ${ }^{10}, 1.6 \%$ were two-part (1/61), 32.8\% three-part (20/61) and 65.6\% four-part (40/61) fractures (Table III). Further assessment showed $60.7 \%(37 / 61)$ of fractures having a calcar-length less than $8 \mathrm{~mm}$ and $42.6 \%$ (26/61) displaying loss of medial hinge integrity on preoperative radiographs.

Each included proximal humerus fracture underwent ORIF through a standardised approach, which typically included beach-chair positioning, perioperative antibiotic prophylaxis and intraoperative image intensification to guide fracture reduction. The deltopectoral approach was utilised

Table I. — Included patient demographics

\begin{tabular}{|l|c|c|c|}
\hline Fracture Type & Female & Male & Total \\
\hline $\begin{array}{l}\text { Number of Patients } \\
\text { (Percentage) }\end{array}$ & $\begin{array}{c}47 \\
(78 \%)\end{array}$ & $\begin{array}{c}13 \\
(12 \%)\end{array}$ & 60 \\
\hline $\begin{array}{l}\text { Mean Age in Years } \\
\text { (SD=Standard } \\
\text { Deviation) }\end{array}$ & $\begin{array}{c}64 \\
(\mathrm{SD}=13)\end{array}$ & $\begin{array}{c}64 \\
(\mathrm{SD}=12.6)\end{array}$ & $\begin{array}{c}64 \\
(\mathrm{SD}=13)\end{array}$ \\
\hline
\end{tabular}

Table III. - Included patient neer fracture classification

\begin{tabular}{|l|c|c|}
\hline Neer Classification & $\begin{array}{c}\text { Number of } \\
\text { Patients }\end{array}$ & Percentage \\
\hline Minimally-Displaced & 0 & $0 \%$ \\
\hline Two-Part & 1 & $1.6 \%$ \\
\hline Three-Part & 20 & $32.8 \%$ \\
\hline Four-Part & 40 & $65.6 \%$ \\
\hline Articular Segment & 0 & $0 \%$ \\
\hline
\end{tabular}

in each case with intraoperative tenodesis of the LHBT at the level of pectoralis major prior to closure. Fracture fixation was attained following reduction through locking plate osteosynthesis in each case, with the DePuy Synthes PHILOS ${ }^{\text {TM }}$ proximal humerus system utilised in $94 \%$ of cases $(57 / 61)$ and the Arthrex Humeral SuturePlate ${ }^{\mathrm{TM}}$ system used in 6\% (4/61). Postoperative rehabilitation in all patients involved radiographic and clinical followup, with a gradual increase in passive and active range of movement in accordance with clinical and radiological review as per a standardised rehabilitation protocol.

Postoperative radiographs utilised for evaluation of evidence ofAVN occurred at a median of 23 months $(\mathrm{SD}=15)$ following the index ORIF procedure. Only 1 patient $(1.6 \%)$ displayed definitive evidence of humeral head AVN (Table IV) following assessment by both independent reviewers. This patient was a 64 year old ASA-I patient presenting with a fourpart proximal humerus fracture with both a calcar length of less than $8 \mathrm{~mm}$ and loss of medial hinge on preoperative radiographs, and underwent fixation using a PHILOS ${ }^{\mathrm{TM}}$ locking plate. She developed postoperative AVN and underwent subsequent total shoulder arthroplasty 12 months following initial ORIF.

Further postoperative radiographic review identified $8.2 \%$ of patients $(5 / 61)$ displaying evidence of tuberosity AVN, with none of these patients

Table II. — Included patient ASA grading

\begin{tabular}{|c|l|c|c|}
\hline ASA Grade & \multicolumn{1}{|c|}{ Definition } & Number of Patients & Percentage \\
\hline ASA I & A normal, healthy patient & 25 & $42 \%$ \\
\hline ASA 2 & A patient with mild systemic disease & 28 & $47 \%$ \\
\hline ASA 3 & A patient with severe systemic disease & 7 & $11 \%$ \\
\hline ASA 4 & A patient with severe systemic disease that is a constant threat to life & 0 & $0 \%$ \\
\hline ASA 5 & A moribund patient not expected to survive without operation & 0 & $0 \%$ \\
\hline
\end{tabular}


Table IV. - Incidence of postoperative radiographic avascular necrosis

\begin{tabular}{|c|c|c|c|c|}
\hline Avascular Necrosis & Number of Patients & Percentage & Number of Reoperations & Percentage Reoperation \\
\hline Humeral Head & 1 & $1.6 \%$ & 1 & $100 \%$ \\
\hline Tuberosity & 5 & $8.2 \%$ & 0 & $0 \%$ \\
\hline
\end{tabular}

requiring additional surgical intervention (Table IV). Additional complications identified showed $1.6 \%$ of included patients (1/61) required early removal of metalwork for loss of fixation position, and $1.6 \%(1 / 61)$ displayed evidence of fracture nonunion at 38 months post-fixation, with neither requiring further surgery.

\section{DISCUSSION}

Proximal humerus fractures are becoming increasingly common (3), and can result in significant impairment, particularly in the elderly patient (8). As a result of this increase, the complications associated with proximal humerus fractures are now more frequently encountered (29), with post-traumatic humeral head AVN of paramount concern in management of these injuries. The exact incidence of post-traumatic AVN in this group has been difficult to quantify precisely, attributed to the heterogeneity in evaluation, treatment and followup (29), with rates estimated to be up to $25 \%$ in three-part fractures $(30,31)$ and range between 0 and $77 \%$ in four-part fractures $(32,33,34)$.

To date, a multitude of risk factors have been described to determine the likelihood of posttraumatic humeral head AVN. While the fracture patterns predisposing patients to post-traumatic proximal humerus AVN are well described $(10,12)$, intraoperative factors are also considered relevant (29). The rationale behind this is based upon the principle that a disruption in blood supply precipitates humeral head AVN. The humeral head's blood supply generally derives from two sources, namely the anterior and posterior circumflex arteries, each arising from the axillary artery. It has been traditionally postulated that the arcuate artery, the termination of the ascending anterolateral branch of the anterior circumflex artery, running laterally to the LHBT makes up the predominant blood supply to the humeral head (35), with the proximal circumflex artery, despite its greater diameter, supplying only the posteroinferior part of the humeral head and posterior greater tuberosity (24). More recent MRI evaluation, however, has suggested the posterior circumflex artery plays a more prominent role than previously suspected, providing up to $64 \%$ of all blood supply to the humeral head (24). As such, preservation of both vascular structures during open reduction and internal fixation (ORIF) is recommended (17).

The versatile deltopectoral approach has been described as a common option for displaced proximal humerus fractures requiring ORIF (36). It generally allows for wide exposure of the surgical field if required, and follows an internervous plane between deltoid and pectoralis major, hence potentially aiding protection of the axillary nerve (17). To aid complex multiple-part fracture visualisation, accurate reduction and optimal fixation, particularly when accessing the head segment through the rotator interval and insuring calcar reduction through this approach (21), we routinely perform a LHBT tenotomy and subsequent tenodesis at the level of pectoralis major. Up to $40 \%$ of post-operative complications following proximal humerus ORIF have been attributed to intraoperative surgical technique (37), and as such, this intraoperative step may be of considerable benefit in helping insure more accurate reduction and fracture fixation, thereby potentially reducing the risk of complications due to surgical technique (37).

Injury to the LHBT has been associated with both shoulder pain and dysfunction (38), and as such an additional benefit to LHBT tenodesis within this context may also exist in regards postoperative pain relief. While the role of the LHBT in humeral stabilisation is suspected to be limited $(39,40)$, pathology to the tendon is an established cause of shoulder pain and dysfunction (41). Significant stromal cell differentiation predisposing the LHBT 
to pathology and resultant pain have been found on histological tendon study following complex proximal humerus fractures (42), and as such it can be postulated that tenodesis of the LHBT during fracture management may negate this potential source of pain as well as minimising the potential risk of scarring or adhesions (43), therefore aiding recovery and return to function. These benefits are further supported by the fact that LHBT tenodesis may minimise potential entrapment of the LHBT at the level of the surgical neck component of the fracture and its associated callus, with LHBT pain having previously been associated with both shoulder pain and locking (44).

While potential benefits to LHBT tenodesis exist, it has been argued that routine tenodesis may predispose the patient to higher risk of humeral head AVN, primarily due to the proximity of the anterolateral branch of both the anterior circumflex artery and its principal arcuate branch to the LHBT in the bicipital groove (24). The risk of iatrogenic arcuate artery injury during surgical fixation has been described as a risk factor for humeral head osteonecrosis (29), and as such potential injury to the arterial supply during LHBT may in theory disrupt humeral head perfusion.

However, our series found radiographic evidence of post-operative humeral head AVN in only $1(1.6 \%)$ patient undergoing LHBT tenodesis at deltopectoral approach, which appears to be an acceptable rate when compared to previously published standards for this fracture pattern [28-32]. This series primarily evaluated displaced three and four part fractures in a predominantly elderly, female population, generally representative of the typical population suffering from proximal humerus fractures $(2,45)$, therefore strengthening the potential safety inference one may make from our findings. In the context of both the potential intraoperative and postoperative advantages discussed previously, a low associated risk of AVN may reinforce the routine use of LHBT tenodesis previously postulated (42).

Our study is a non-randomised retrospective study of a relatively small sample size, which can limit the clinical significance of its findings. The small sample size and retrospective study design also contributed to the inability to perform a power analysis and comparative statistics which precludes the ability of this study to evaluate for a potentially statistically insignificant change in the rate of AVN following LHBT tenodesis. This may be further exacerbated by the varying length of post-operative radiographic follow-up, as well as the lack of correlation of plain radiographic evidence with both clinical and more advanced radiographic evidence of humeral head AVN. Despite these limitations, we believe this study to be of benefit as it highlights the relative safety of intraoperative LHBT tenodesis in the context of postoperative AVN. Plain radiographic evidence of AVN of the femoral head has been shown to occur at from one year following onset of symptoms (46), and extrapolation of this to the proximal humerus entails that the median radiographic follow-up of 23 months may be of satisfactory length to allow potential AVN to become apparent on plain film. As such we feel this paper offers some initial evidence for what can be a useful intraoperative technique during proximal humerus ORIF. Ideally, randomised studies including both clinical and advanced radiographic follow-up of postoperative patients would further help evidence the safety of this intraoperative technique.

In conclusion, our experience has shown that intraoperative tenodesis of the LHBT during deltopectoral approach to proximal humerus ORIF does not appear to be associated with a high rate of humeral head AVN, even in complex, multiple part proximal humerus fractures, adding weight to a technique which may theoretically aid in both fracture fixation and postoperative pain reduction.

\section{REFERENCES}

1. Horak J, Nilsson BE. Epidemiology of fracture of the upper end of the humerus. Clinical orthopaedics and related research. 1975 ; (112) : 250-3.

2. Palvanen M, Kannus P, Niemi S, Parkkari J. Update in the epidemiology of proximal humeral fractures. Clinical orthopaedics and related research. 2006 ; $442: 87-92$.

3. Roux A, Decroocq L, El Batti S, et al. Epidemiology of proximal humerus fractures managed in a trauma center. Orthopaedics \& traumatology, surgery \& research : OTSR. 2012 ; 98(6) : 715-9.

4. Chu SP, Kelsey JL, Keegan TH, et al. Risk factors for proximal humerus fracture. American journal of epidemiology. $2004 ; 160(4)$ : 360-7. 
5. Court-Brown CM, Garg A, McQueen MM. The epidemiology of proximal humeral fractures. Acta orthopaedica Scandinavica. $2001 ; 72(4): 365-71$.

6. Vachtsevanos L, Hayden L, Desai AS, Dramis A. Management of proximal humerus fractures in adults. World journal of orthopedics. $2014 ; 5(5)$ : 685-93.

7. Launonen AP, Lepola V, Saranko A, et al. Epidemiology of proximal humerus fractures. Archives of osteoporosis. $2015 ; 10: 209$.

8. Kim SH, Szabo RM, Marder RA. Epidemiology of humerus fractures in the United States: nationwide emergency department sample, 2008. Arthritis care \& research. $2012 ; 64(3):$ 407-14.

9. Khmelnitskaya E, Lamont LE, Taylor SA, et al. Evaluation and management of proximal humerus fractures. Advances in orthopedics. 2012;2012 : 861598.

10. Neer CS, 2nd. Displaced proximal humeral fractures. I. Classification and evaluation. The Journal of bone and joint surgery, American volume. 1970 ; 52(6) : 1077-89.

11. Meinberg E AJ, Roberts $\mathbf{C}$, et al. Fracture and Dislocation Classification Compendium - 2018. Journal of Orthopaedic Trauma. 32(Number 1 ; Supplement).

12. Hertel R, Hempfing A, Stiehler M, Leunig M. Predictors of humeral head ischemia after intracapsular fracture of the proximal humerus. Journal of shoulder and elbow surgery. 2004 ; 13(4) : 427-33.

13. Kontakis G, Koutras C, Tosounidis T, Giannoudis P. Early management of proximal humeral fractures with hemiarthroplasty : a systematic review. The Journal of bone and joint surgery, British volume. 2008 ; 90(11) : 1407-13.

14. Gaebler C, McQueen MM, Court-Brown CM. Minimally displaced proximal humeral fractures : epidemiology and outcome in 507 cases. Acta orthopaedica Scandinavica. $2003 ; 74(5): 580-5$.

15. Schumaier A, Grawe B. Proximal Humerus Fractures : Evaluation and Management in the Elderly Patient. Geriatric Orthopaedic Surgery \& Rehabilitation. 2018; 9: 2151458517750516.

16. Mauro CS. Proximal humeral fractures. Current Reviews in Musculoskeletal Medicine. 2011 ; 4(4) : 214-20.

17. Berkes MB, Little MTM, Lorich DG. Open reduction internal fixation of proximal humerus fractures. Current Reviews in Musculoskeletal Medicine. 2013 ; 6(1) : 47-56.

18. Archer LA, Furey A. Rate of avascular necrosis and time to surgery in proximal humerus fractures. Musculoskeletal surgery. $2016 ; 100(3)$ : 213-6.

19. Carofino BC, Leopold SS. Classifications in brief: the Neer classification for proximal humerus fractures. Clinical orthopaedics and related research. 2013 ; 471(1) : 39-43.

20. Menendez ME, Ring D, Heng M. Proximal humerus fracture with injury to the axillary artery : a populationbased study. Injury. $2015 ; 46(7)$ : 1367-71.

21. Robinson CM, Murray IR. The extended deltoidsplitting approach to the proximal humerus : variations and extensions. The Journal of bone and joint surgery, British volume. 2011 ; 93(3) : 387-92.
22. Gregory TM, Vandenbussche E, Augereau B. Surgical treatment of three and four-part proximal humeral fractures. Orthopaedics \& traumatology, surgery \& research : OTSR. 2013 ; 99(1 Suppl) : S197-207.

23. Aflatooni JO, Meeks BD, Froehle AW, Bonner KF. Biceps tenotomy versus tenodesis : patient-reported outcomes and satisfaction. Journal of Orthopaedic Surgery and Research. $2020 ; 15(1): 56$.

24. Gerber C, Schneeberger AG, Vinh TS. The arterial vascularization of the humeral head. An anatomical study. The Journal of bone and joint surgery, American volume. 1990 ; 72(10) : 1486-94.

25. Keough N, de Beer T, Uys A, Hohmann E. An anatomical investigation into the blood supply of the proximal humerus : surgical considerations for rotator cuff repair. JSES Open Access. 2019 ; 3(4) : 320-7.

26. Sankar A, Johnson SR, Beattie WS, Tait G, Wijeysundera DN. Reliability of the American Society of Anesthesiologists physical status scale in clinical practice. British journal of anaesthesia. 2014 ; 113(3) : 424-32.

27. Ficat RP AJ. Forage-biopsie de la tete femorale dans I'osteonecrose primative. Observations histo-pathologiques portant sur huit forages. Rev Rhum. 1964 ; 31 : 25764.

28. Ficat RP. Idiopathic bone necrosis of the femoral head. Early diagnosis and treatment. The Journal of bone and joint surgery, British volume. $1985 ; 67(1): 3-9$.

29. Patel S, Colaco HB, Elvey ME, Lee MH. Post-traumatic osteonecrosis of the proximal humerus. Injury. 2015 ; 46(10) : 1878-84.

30. Esser RD. Treatment of three- and four-part fractures of the proximal humerus with a modified cloverleaf plate. $J$ Orthop Trauma. 1994 ; 8(1): 15-22.

31. O. Hagg BJL. Aspects of prognostic factors in comminuted and dislocated proximal humeral fractures. JE Bateman, RP Welsh (Eds), Surgery of the shoulder, BC Decker, Philadelphia (1984). 1984.

32. Schai P, Imhoff A, Preiss S. Comminuted humeral head fractures : A multicenter analysis. Journal of shoulder and elbow surgery. $1995 ; 4(5): 319-30$.

33. Lee CK, Hansen HR. Post-traumatic avascular necrosis of the humeral head in displaced proximal humeral fractures. The Journal of trauma. $1981 ; 21(9)$ : 788-91.

34. Sturzenegger M, Fornaro E, Jakob RP. Results of surgical treatment of multifragmented fractures of the humeral head. Archives of orthopaedic and traumatic surgery Archiv fur orthopadische und Unfall-Chirurgie. 1982; 100(4) : 24959.

35. Laing PG. The arterial supply of the adult humerus. The Journal of bone and joint surgery, American volume. $1956 ; 38-\mathrm{a}(5)$ : 1105-16.

36. Buecking B, Mohr J, Bockmann B, Zettl R, Ruchholtz S. Deltoid-split or Deltopectoral Approaches for the Treatment of Displaced Proximal Humeral Fractures? Clinical ortho-paedics and related research. 2014 ; 472(5) : 157685. 
37. Sudkamp N, Bayer J, Hepp P, et al. Open reduction and internal fixation of proximal humeral fractures with use of the locking proximal humerus plate. Results of a prospective, multicenter, observational study. The Journal of bone and joint surgery, American volume. 2009 ; 91(6) : 1320-8.

38. AlQahtani SM, Bicknell RT. Outcomes following long head of biceps tendon tenodesis. Current reviews in musculoskeletal medicine. 2016 ; 9(4) : 378-87.

39. Ahrens PM, Boileau P. The long head of biceps and associated tendinopathy. The Journal of bone and joint surgery, British volume. 2007 ; 89(8) : 1001-9.

40. Sethi N, Wright R, Yamaguchi K. Disorders of the long head of the biceps tendon. Journal of shoulder and elbow surgery. $1999 ; 8(6): 644-54$.

41. Ge Ha, Zhang Q, Sun Y, et al. Tenotomy or Tenodesis for the Long Head of Biceps Lesions in Shoulders: A Systematic Review and Meta-Analysis. PLOS ONE. 2015 ; 10(3) : e0121286.
42. Tosounidis T, Hadjileontis C, Georgiadis M, Kafanas A, Kontakis G. The tendon of the long head of the biceps in complex proximal humerus fractures: a histological perspective. Injury. 2010 ; 41(3) : 273-8.

43. Sinha S, Kelly CP. Fixed angle locking plates for proximal humeral fracture fixation. Ann R Coll Surg Engl. 2010 ; 92(8) : 631-4.

44. Boileau P, Ahrens PM, Hatzidakis AM. Entrapment of the long head of the biceps tendon : the hourglass biceps-a cause of pain and locking of the shoulder. Journal of shoulder and elbow surgery. 2004 ; 13(3) : 249-57.

45. Bergdahl C, Ekholm C, Wennergren D, Nilsson F, Möller M. Epidemiology and patho-anatomical pattern of 2,011 humeral fractures : data from the Swedish Fracture Register. BMC Musculoskelet Disord. 2016; 17 : 159.

46. Stoica Z, Dumitrescu D, Popescu M, et al. Imaging of avascular necrosis of femoral head : familiar methods and newer trends. Curr Health Sci J. 2009 ; 35(1) : 23-8. 